\title{
A REMARK ON THE LOCAL WELL-POSEDNESS FOR A COUPLED SYSTEM OF MKDV TYPE EQUATIONS IN $H^{s} \times H^{k}$
}

\author{
XAVIER CARVAJAL
}

Abstract. We consider the initial value problem associated to a system consisting modified Korteweg-de Vries type equations

$$
\begin{cases}\partial_{t} v+\partial_{x}^{3} v+\partial_{x}\left(v w^{2}\right)=0, & v(x, 0)=\phi(x), \\ \partial_{t} w+\alpha \partial_{x}^{3} w+\partial_{x}\left(v^{2} w\right)=0, & w(x, 0)=\psi(x),\end{cases}
$$

and using only bilinear estimates of the type $\left\|J^{\gamma} F_{b_{1}}^{1} \cdot J^{\beta} F_{b_{2}}^{2}\right\|_{L_{x}^{2} L_{t}^{2}}$, where $J$ is the Bessel potential and $F_{b_{j}}^{j}, j=1,2$ are multiplication operators, we prove the local well-posedness results for given data in low regularity Sobolev spaces $H^{s}(\mathbb{R}) \times H^{k}(\mathbb{R})$ for $\alpha \neq 0,1$. In this work we improve the previous result in [6], extending the LWP region from $|s-k|<1 / 2$ to $|s-k|<1$. This result is sharp in the region of the LWP with $s \leqslant 0$ and $k \leqslant 0$, in the sense of the trilinear estimates fails to hold.

Mathematics subject classification (2010): 35Q35, 35Q53.

Keywords and phrases: Korteweg-de Vries equation, Cauchy problem, local well-posedness.

\section{REFERENCES}

[1] M. Ablowitz, D. Kaup, A. Newell and H. Segur, Nonlinear evolution equations of physical significance, Phys. Rev. Lett. 31 (2) (1973) 125-127.

[2] E. Alarcon, J. Angulo And J. F. Montenegro, Stability and instability of solitary waves for a nonlinear dispersive system, Nonlinear Analysis 36 (1999) 1015-1035.

[3] B. Alvarez, X. CARVAJal, On the local well-posedness for some systems of coupled KdV equations, Nonlinear Analysis 69, (2008) 692-715.

[4] D. BeKiranov, T. OGAWA, G. Ponce, Weak solvability and well-posedness of a coupled Schrödinger-Korteweg de Vries equation for capillary-gravity wave interactions, Proceedings of the American Mathematical Society 125 (1997) 2907-2919.

[5] J. Bourgain, Fourier transform restriction phenomena for certain lattice subsets and applications to nonlinear evolution equations, II, The KdV-equation, Geom. Funct. Anal. 3 (1993) 209-262.

[6] X. CARVAJAL, L. Esquivel, R. SAntos, Sharp well-posedness for a coupled system of mkdv type equations, (2020) arXiv:2003.12619v1.

[7] X. Carvajal, M. PantheE, Sharp well-posedness for a coupled system of mkdv type equations, J. Evol. Equ. 19, (2019), 1167-1197.

[8] X. CARVAJAL, Sharp global well-posedness for a higher order Schrödinger equation, J. Fourier Anal. Appl. 12 (2006) no. 1, 53-70.

[9] X. CARVAJAL, Local well-posedness for a higher order nonlinear Schrödinger equation in Sobolev spaces of negative indices, Electron. J. Differential Equations 13 (2004) 1-13.

[10] M. Chen, B. Guo, Local Well and Ill Posedness for the Modified KdV Equations in Subcritical Modulation Spaces, (2018) arXiv:1811.05182.

[11] M. Christ, J. Holmer, D. TATARU, Low regularity a priori bounds for the modified Korteweg-de Vries equation, Libertas Mathematica 32 (2012) no. 1 51-75.

[12] A. Corcho, M. PAntheE, Global well-posedness for a coupled modified KdV system, Bull Braz Math Soc, New Series 43, (2012) 27-57. 
[13] J. Ginibre, Y. Tsutsumi, G. Velo, On the Cauchy problem for the Zakharov system, J. Funct. Anal. 151 (1997), no. 2, 384-436.

[14] Z. Guo, Global well-posedness of Korteweg-de Vries equation in $H^{-3 / 4}(\mathbb{R})$, J. Math. Pures Appl. 91 (2009) 583-597.

[15] T. Он, Diophantine conditions in well-posedness theory of coupled KdV-type systems: local theory, Int. Math. Res. Not. IMRN (2009), no. 18, 3516-3556.

[16] C. E. Kenig, G. Ponce, L. Vega, A bilinear estimate with applications to the KdV equation, J. Amer. Math. Soc. 92 (1996) 573-603.

[17] C. E. Kenig, G. Ponce, L. Vega, Well-posedness and scattering results for the generalized Korteweg-de Vries equation via the contraction principle, Comm. Pure Appl. Math. 46 (1993) 527620.

[18] C. E. Kenig, G. Ponce, L. Vega, The Cauchy problem for the Korteweg-de Vries equation in Sobolev spaces of negative indices, Duke Math. J. 71 (1993), no. 1, 1-21.

[19] R. Killip, M. Vis AN, KdV is well-posed in $H^{-1}$, Ann. of Math. 190, no. 1 (2019) 249-305.

[20] Noвu Kisнiмото, Well-posedness of the Cauchy problem for the Korteweg-de Vries equation at the critical regularity, Differential Integral Equations 22 (2009), no. 5-6, 447-464.

[21] T. TAO, Multilinear weighted convolution of $L^{2}$-functions, and applications to nonlinear dispersive equations, Amer. J. Math. 123 (2001) no. 5, 839-908.

[22] J. F. Montenegro, Sistemas de equações de evolução não lineares; Estudo local, global e estabilidade de ondas solitarias, Ph. D. Thesis, IMPA, Rio de Janeiro (1995).

[23] H. TAKAOKA, Well-posedness for the higher order nonlinear Schrödinger equation, Adv. Math. Sci. Appl. 10 (2000) 149-171. 